\title{
Integration of predictive-corrective incompressible SPH and Hodgkin-Huxley based models in the OpenWorm in silico model of C. elegans
}

\author{
Michael Vella ${ }^{1 *}$, Andrey Palyanov ${ }^{2}$, Padraig Gleeson ${ }^{3}$, Sergey Khayrulin ${ }^{2}$ \\ From Twenty Second Annual Computational Neuroscience Meeting: CNS*2013 \\ Paris, France. 13-18 July 2013
}

OpenWorm is an international collaboration with the aim of producing an integrative computational model of Caenorhabditis elegans to further the understanding of how macroscopic behaviour of the organism emerges from aggregated biophysical processes. A core component of the project involves the integration of electrophysiological modelling and predictive-corrective incompressible smoothed particle hydrodynamics (PCISPH) to model how neuronal and muscle dynamics effect the nematode's behaviour. Several tools are being utilised and developed in the course of the project:

- Electrophysiological model parameters are constrained to reproduce experimental measurements using the Optimal Neuron toolkit [1]

- A PCISPH solver is under development [2] - a combination of general PCISPH algorithms proposed by [3], boundary-handling algorithms proposed by [4], a surface tension model based on [5] and our own implementation of elastic matter and biophysicsspecific features, as well as parallelization, optimization and tuning. It is the first open source, parallel OpenCL/ $\mathrm{C}++$ PCISPH high-performance implementation.

- A generic model integration framework (Gepetto [6]) will be used to integrate electrophysiology and bodywall interactions

- All electrophysiological models are NeuroMLcompatible [7].

\footnotetext{
* Correspondence: mv333@cam.ac.uk

'Department of Physiology, Development and Neuroscience, University of Cambridge, Cambridge, CB2 3DY, UK

Full list of author information is available at the end of the article
}

- The Open Worm Browser provides a powerful way to visualise C. Elegans anatomy [8]

All of the above mentioned applications are open source, freely available and can be used for modelling other neuronal systems.

\section{Author details}

'Department of Physiology, Development and Neuroscience, University of Cambridge, Cambridge, CB2 3DY, UK. ${ }^{2}$ Lab. of complex systems simulations, A.P. Ershov Institute of Informatics Systems, Siberian Branch of the Russian Academy of Sciences, Novosibirsk, 630090, Russian Federation. ${ }^{3}$ Department of Neuroscience, Physiology and Pharmacology, University College London, London, UK

Published: 8 July 2013

\section{References}

1. Optimal Neuron Toolkit. [https://github.com/vellamike/Optimal-Neuron].

2. Open Worm PCISPH Simulator. [https://github.com/openworm/SmoothedParticle-Hydrodynamics].

3. Solenthaler B, Pajarola R: Predictive-corrective incompressible SPH. ACM Trans Graph 2009, 28(3).

4. Inmsen M, Akinci N, Gissler M, Teschner M: Boundary Handling and Adaptive Time-stepping for PCISPH. Proc VRIPHYS Copenhagen, Denmark; 2010, 79-88, Nov 11-12.

5. Becker M, Teschner M: Weakly compressible SPH for free surface flows. Proceedings of the 2007 ACM SIG-GRAPH/Eurographics 2007.

6. Geppetto Simulation Engine. [https://github.com/openworm/OpenWorm/ wiki/Geppetto-Overview]

7. Gleeson P, Crook S, Cannon RC, Hines ML, Billings GO, et al: NeuroML: A Language for Describing Data Driven Models of Neurons and Networks with a High Degree of Biological Detail. PLoS Comput Biol 2010, 6(6): e1000815, doi:10.1371/journal.pcbi.1000815.

8. Open Worm Browser. [http://browser.openworm.org/].

doi:10.1186/1471-2202-14-S1-P209

Cite this article as: Vella et al:: Integration of predictive-corrective incompressible SPH and Hodgkin-Huxley based models in the OpenWorm in silico model of C. elegans. BMC Neuroscience 201314 (Suppl 1):P209.

\section{() Biomed Central}

